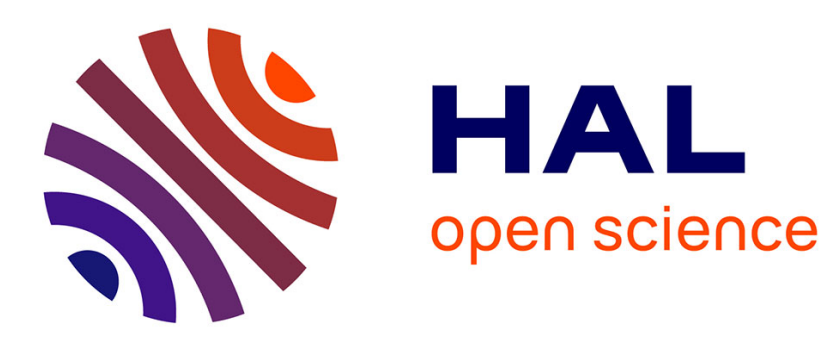

\title{
Influence des systèmes de référence sur les profils de collaboration dans un environnement virtuel
}

Amine Chellali, Isabelle Milleville-Pennel, Cédric Dumas

\section{To cite this version:}

Amine Chellali, Isabelle Milleville-Pennel, Cédric Dumas. Influence des systèmes de référence sur les profils de collaboration dans un environnement virtuel. IHM 2008, Sep 2008, Metz, France. pp.63-70. hal-00470432

\section{HAL Id: hal-00470432 \\ https://hal.science/hal-00470432}

Submitted on 20 May 2010

HAL is a multi-disciplinary open access archive for the deposit and dissemination of scientific research documents, whether they are published or not. The documents may come from teaching and research institutions in France or abroad, or from public or private research centers.
L'archive ouverte pluridisciplinaire HAL, est destinée au dépôt et à la diffusion de documents scientifiques de niveau recherche, publiés ou non, émanant des établissements d'enseignement et de recherche français ou étrangers, des laboratoires publics ou privés. 


\section{Influence des systèmes de référence sur les profils de collaboration dans un environnement virtuel}

\author{
Amine Chellali \\ EMN - IRCCyN \\ 4 rue $A$. Kastler \\ 44307 Nantes CEDEX 3 \\ amine.chellali@emn.fr
}

\author{
Isabelle Milleville-Pennel \\ CNRS - IRCCyN \\ 1, rue de la Noë - BP 92101 \\ 44321 Nantes CEDEX 03 \\ isabelle.milleville- \\ pennel@irccyn.ec-nantes.fr
}

\author{
Cedric Dumas \\ EMN - IRCCyN \\ 4 rue $A$. Kastler \\ 44307 Nantes CEDEX 3 \\ cedric.dumas@emn.fr
}

\begin{abstract}
RESUMÈ
Les Environnements Virtuels Collaboratifs sont des espaces 3D partagés dans lesquels les personnes travaillent ensemble. Pour que la collaboration soit efficace, les utilisateurs doivent avoir des compréhensions compatibles du monde virtuel. Dans cette étude exploratoire nous avons manipulé le contenu de l'environnement virtuel partagé pour comprendre comment les utilisateurs construisent verbalement des représentations communes de cet environnement. Les résultats montrent que la présence d'un indice visuel fixe dans l'environnement virtuel influence la façon dont les opérateurs collaborent.
\end{abstract}

MOTS CLES : Environnement virtuel, collaboration, référentiel commun, Interface 3D, contexte partagé.

\begin{abstract}
Virtual collaborative environment are 3D shared spaces in which people can work together. To collaborate through these systems users must have a shared comprehension of the environment. In this study we manipulated contents of a virtual environment to understand how people construct compatible comprehensions of this shared environment. Results show that the use of stable visual landmarks leads to different collaboration profiles.
\end{abstract}

CATEGORIES AND SUBJECT DESCRIPTORS: H.5.1 Multimedia Information Systems. H.5.3 Group and Organization Interfaces

GENERAL TERMS: Experimentation, Human Factors.

KEY WORDS: Virtual environments, Collaboration, Common frame of reference, 3D, common ground.

\section{INTRODUCTION}

Dans une situation de collaboration dans le monde réel, les opérateurs construisent et entretiennent des représen- tations mentales compatibles de l'environnement. Une représentation mentale est l'image qu'un individu se fait d'une situation par la pensée. Ces représentations doivent être similaires entre les utilisateurs dans une même situation collaborative pour limiter les incompréhensions dans le dialogue et favoriser la réalisation des objectifs. Pour ce faire, les partenaires échangent des informations via plusieurs modalités sensorielles (visuelle, auditive et haptique). Cependant, dans un Environnement Virtuel (EV), les canaux de communication sont restreints, notamment lorsque les opérateurs sont distants. C'est pourquoi l'EV doit offrir d'autres informations pour construire un Référentiel Commun (RC) efficient entre partenaires: ce dernier est constitué des représentations communes des actions à effectuer sur les objets de l'EV ainsi que de l'organisation spatiale de ces objets.

Dans cet article, nous définirons d'abords certains concepts importants : les Environnement Virtuel Collaboratif (EVC) et la construction d'un RC dans une situation de collaboration. Nous discuterons ensuite, des systèmes de référence que les opérateurs utilisent pour construire ce RC. Dans la seconde partie, nous présenterons une étude expérimentale concernant l'impact des indices contextuels sur la construction d'un RC dans l'EV et sur les profils de collaboration qui peuvent en résulter.

\section{ENVIRONNEMENTS VIRTUELS COLLABORATIFS}

Les EVC sont des espaces numériques dans lesquels des utilisateurs peuvent se rencontrer, partager des objets virtuels et travailler ensemble [4]. Ils sont utilisés dans l'apprentissage, la CAO ou encore les applications ludoéducatives. Les EVC permettent de réduire les coûts et les risques liés à certaines applications (médicales, spatiales,...). Cependant, la principale difficulté reste de construire des EV qui supportent la collaboration.

\section{COLLABORATION ET REFERENTIEL COMMUN}

Dans une situation de collaboration, il existe une symétrie dans les interactions entre des partenaires qui ont souvent un but commun et qui travaillent ensemble [6]. Elle implique une interaction entre les participants, une communication synchrone et de la négociation. Elle ne se limite pas à une tâche partagée. En effet, pour comprendre leurs intentions d'actions mutuelles, les participants doivent définir et entretenir chacun une représenta- 
tion de la situation compatible avec celles des autres. Celle-ci constitue leur RC [11]. Ce dernier facilite la compréhension des communications entre les opérateurs, c'est le cas par exemple de «l'humour de connivence », qui empêche généralement (par défaut de RC) les noninitiés de comprendre «une blague privée». Le RC permet à des opérateurs d'agir simultanément avec des actions différentes mais complémentaires. Par exemple lors d'une opération chirurgicale, les membres de l'équipe médicale réalisent chacun une tâche différente tout en anticipant les actions des autres pour éviter toute interférence.

Cette notion de RC rejoint d'autres concepts définis dans la littérature tels que le contexte partagé (Common ground) [5], la modélisation mutuelle (mutual modelling) [6] et l'espace commun d'un problème (joint problem space) [13]. Dans toutes ces définitions, l'accent est mis sur la construction et le maintient d'une représentation commune dans le but d'avoir des décisions compatibles entres les opérateurs. Chaque participant à l'activité collaborative doit donc confronter ses propres représentations à celles des autres en prenant conscience des divergences qui peuvent exister et de la possibilité de les éliminer. Cette confrontation continue est un processus actif de communication qui consiste pour chaque opérateur à faire des efforts de recherche d'indices de compréhension chez le partenaire et à lui fournir ses propres indices de compréhension (le processus de grounding défini par Clark et Brennan [5]). La communication est donc le moteur de la collaboration et elle peut avoir deux formes : explicite (verbale ou écrite) et implicite (la gestuelle, les expressions faciales,...). Pour que la communication soit efficace dans une situation collaborative, les efforts fournis par les opérateurs pour maintenir leur RC doivent être minimisés (le moindre effort collaboratif) [5]. Pour ce faire, les opérateurs doivent exploiter les propriétés de leur environnement: l'organisation spatiale commune des objets, la construction des référentiels à partir des connaissances communes du monde qui les entoure (exemple : «au feu tourner à gauche »), la proximité des partenaires et des objets de l'interaction, le sentiment de présence des partenaires dans l'environnement et la conscience de leurs activités courantes [7].

\section{PROBLÈMES LIÉS AUX EVC}

Dans le monde réel, les propriétés décrites précédemment permettent aux opérateurs d'organiser leurs comportements pour mieux collaborer. Cependant, du fait des caractéristiques propres aux EV, certaines propriétés deviennent inexploitables directement : les opérateurs ne partagent plus forcement les mêmes points de vue, les personnes avec lesquelles ils interagissent ne sont pas à proximité, le partage d'informations concernant l'activité de l'autre est limité, les objets avec lesquels ils interagissent ne sont plus à proximité. Cela peut entraver la communication entre les opérateurs. Par exemple, des informations référentielles de type «gauche/droite»ne sont pas pertinentes suivant le point de vue. De plus, la communication dans l'EV est souvent réduite à sa composante explicite. En effet, la gestuelle et les expressions faciales sont rarement reproduites dans un EV. Ce qui implique une nouvelle forme d'interaction collaborative qui doit s'adapter aux spécificités des EV.

Les problèmes liés à la compréhension mutuelle sont de plus en plus abordés dans la littérature : Kraut et al. [10] ont montré que le partage d'un espace visuel entraine davantage de pointage et d'expressions déictiques (celui là, par là) et permet ainsi d'améliorer la collaboration. Axelsson et al. [1] ont comparé la construction d'un contexte partagé dans un EVC selon le degré d'immersion des utilisateurs. Les résultats ont montré que ces derniers tentaient de trouver la meilleure stratégie permettant de réduire le «coût» de la communication. L'étude de Hindmarsh et $\mathrm{Al}$. [8] portait sur les problèmes liés aux différences de points de vue qui peuvent induire des incompréhensions et ainsi gêner la collaboration (à cause de l'ignorance de chaque opérateur des possibilités et des contraintes du partenaire). La verbalisation dans ce cas n'est plus suffisante et la référenciation (action de référencer) des objets devient une activité à part entière. Les auteurs proposent d'inclure des indices sur l'activité des utilisateurs pour pallier à ces problèmes. Spante et al. [14] ont montré d'un autre coté, que la connaissance des contraintes et capacités du partenaire permet un meilleur partage des tâches et donc une meilleure collaboration.

Ces résultats montrent que le partage d'informations visuelles est nécessaire pour réduire le coût des communications dans une situation collaborative. Cependant, il est nécessaire de les enrichir avec des indices collaboratifs pour améliorer la construction d'un RC. Par exemple, renseigner son partenaire sur sa propre activité peut améliorer la construction du RC. Enfin, les EVC doivent tenir compte des différents systèmes de référence utilisés par les opérateurs pour coder les informations spatiales afin de faciliter les échanges entre eux (voir la section suivante). En effet, quand ces derniers ne partagent pas un même point de vue, mettre en valeur leurs connaissances communes de l'environnement (les positions relatives des objets entre eux par exemple) peut réduire les problèmes d'incompréhensions des intentions d'actions.

\section{SYSTEMES DE REFERENCE}

\section{Construction d'un Système de Référence}

La spécification des actions d'un opérateur sur les objets de son environnement est étroitement liée à son point de vue et à la disponibilité des indices contextuels. Elle peut se faire sur la base de deux systèmes [3] :

(a) un système de référence égocentré dans lequel l'opérateur se base sur son corps ou certaines parties de son corps pour planifier ses actions. Ce système est privilégié lorsqu'il s'agit d'effectuer une action, 
comme saisir un objet, effectuer une rotation mentale, ou viser une cible.

(b) un système de référence exocentré dans lequel l'opérateur se base sur des éléments présents dans l'environnement. Il permet de créer ou accéder à une représentation, lors d'une description spatiale d'une pièce ou encore la lecture d'une carte indépendamment de son propre point de vue. Il nécessite en contrepartie de faire des rotations mentales lorsque l'on veut faire correspondre les positions des objets à son propre point de vue.

Un opérateur peut aussi utiliser un système exocentré relatif. En effet, il peut spécifier une action par rapport à un objet mais cette référenciation n'aura de signification que si elle est rapportée à son point de vue (par exemple, la phrase «mettre le stylo derrière le livre » n'aura de signification que si l'opérateur est devant le livre).

\section{Quel Système de Référence dans un EVC}

Dans une situation de collaboration, les operateurs utilisent les systèmes de référence pour expliciter leurs actions à leur partenaire. En dépit de l'importance des systèmes de référence dans les EVC et leur influence sur la construction du RC, peu de recherches abordent ce problème. Cependant, les résultats de certains travaux nous ont permis de poser nos hypothèses.

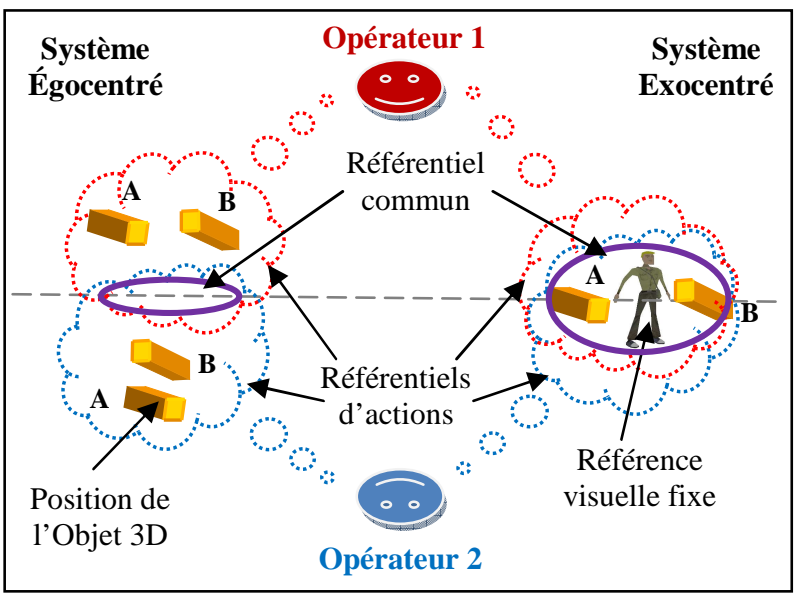

Figure 1 : utilisation des systèmes de référence dans un EV, dans un système égocentré, pour l'opérateur 1, l'objet A se trouve sur sa droite et l'objet $B$ sur sa gauche alors que pour l'opérateur 2, l'objet A se trouve devant et l'objet B se trouve derrière. Dans un système exocentré, pour les deux opérateurs, l'objet A se trouve sur la droite de la référence fixe (représentée par un personnage virtuel) et l'objet B sur sa gauche.

Roberts [12] a montré que dans les environnements 3D, les opérateurs tendent à utiliser un système de référence égocentré. Cependant dans une activité collaborative, du fait de la divergence des points de vue des opérateurs sur la scène, la spécification des actions dans un système égocentré (propre à chaque opérateur) peut induire des incompréhensions et/ou des actions antagonistes. De plus, dans un EV contenant des objets similaires, il est difficile de référencer ces derniers sans ambigüités (cf.
Figure 1). Ceci complique la tâche de construction du RC et gêne l'activité collaborative.

D'autres travaux ont montré que l'utilisation du système de référence exocentré est liée à la présence des indices contextuelle dans l'espace d'action [2]. On peut donc supposer que lorsque la situation devient suffisamment complexe (points de vus différents, manipulations multiples de l'objet ou de ses parties...) la présence de ces indices contextuels incite les opérateurs à se servir naturellement de ce système de référence pour construire leur RC. Il peut induire une meilleure coordination pour la planification des actions et réduire les ambiguïtés liées à la référenciation des objets. De plus, le genre des opérateurs peut aussi influencer la construction du RC. En effet, l'utilisation d'un système exocentré nécessite d'effectuer des rotations mentales. Ainsi, les opérateurs masculins devraient utiliser ce système plus spontanément que les opérateurs féminin. En effet, Kimura [9] observe que les hommes et les femmes ont des aptitudes spatiales différentes. Plusieurs tests ont montré des avantages en faveur des hommes spécialement en ce qui concerne les rotations mentales. Les hommes utilisent le plus souvent les propriétés géométriques de l'espace pour se repérer. Les femmes de leur coté sont plus douées que les hommes pour se souvenir des repères sur un chemin ou pour se souvenir des positions des objets dessinés sur le papier. Ces différences peuvent s'expliquer par l'utilisation de systèmes de références différents. Ceci peut induire des stratégies différentes entre les hommes et les femmes pour des tâches de manipulation d'objets 3D.

Dans cette étude exploratoire, nous nous intéresserons au problème du choix par les opérateurs d'un système de référence particulier lorsqu'ils décrivent leurs actions sur des objets virtuels.

Plus précisément, en étudiant les stratégies spontanées de collaboration entre les opérateurs, l'objectif est de :

- Montrer l'importance des références spatiales fixes dans l'espace d'action permettant de situer les objets entre eux et non plus uniquement par rapport à l'opérateur.

- Montrer l'impact du genre des participants sur le choix de leur système de référence et la construction de leur $\mathrm{RC}$.

- Montrer les impacts possibles de ces référentiels sur les profils de collaboration qui peuvent s'observer entre les opérateurs.

\section{HYPOTHESES}

- H1. Les opérateurs utilisent davantage le système de référence exocentré et exocentré relatif en présence d'un référentiel spatial fixe.

- H2. L'utilisation du système exocentré est moins évidente pour les opérateurs féminins. 
- H3. L'utilisation d'un système de référence exocentré permet d'avoir un RC plus vaste entre les opérateurs.

- H4. Le système de référence utilisé (en élargissant le $\mathrm{RC}$ ) influence les formes de collaboration qui se développent entre les opérateurs.

\section{METHODE}

\section{Sujets}

44 participants (âgés de 20 à 27 ans) étaient répartis en 22 binômes (10 binômes de filles et 12 binômes de garçons). Aucun sujet n'avait de connaissance préalable de l'EV. Les sujets de chaque binôme ne se connaissaient pas entre eux avant l'expérience. Le niveau d'expertise des participants (mesuré par leur fréquence d'utilisation des jeux vidéo et/ou modeleurs 3D sur une échelle à 5 échelons) était équivalent dans chaque groupe.

\section{Matériel}

Les dispositifs d'entrée choisis étaient des manettes de jeu de type Joypad à 6 degrés de liberté (3 DdL pour les translations, 2 DdL pour les rotations et $1 \mathrm{DdL}$ pour manipuler le point de vue sur la scène).

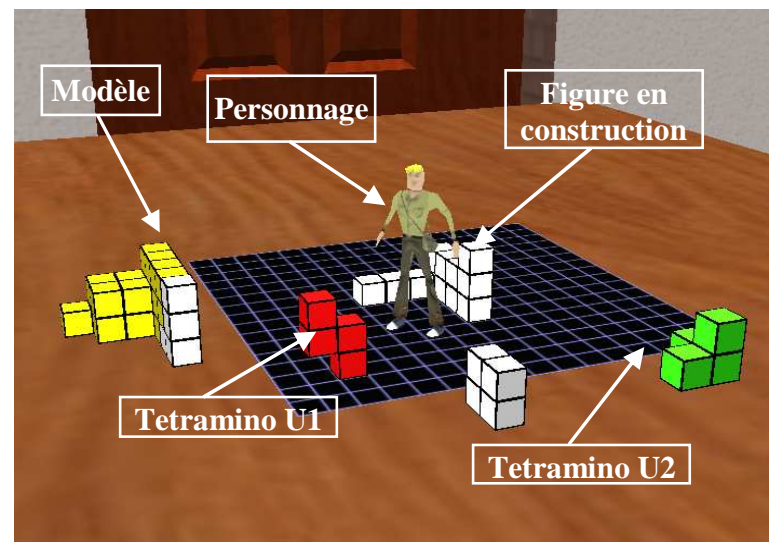

Figure 2 : Environnement virtuel

\section{Interface 3D}

L'EV a été conçu sous l'environnement de développement Virtools. L'EV consistait en une table sur laquelle étaient posés 6 tétraminos ${ }^{1}$ de la même couleur (Figure 2). Les opérateurs pouvaient interagir avec ceux-ci en utilisant les manettes de jeu. Deux opérateurs pouvaient déplacer chacun une pièce différente en même temps.

Référence spatiale fixe. Selon la condition expérimentale (voir la section Variables ci-après) un personnage virtuel représentant une Référence Spatiale Fixe (RSF) était placé au centre de la scène (Figure 2). Le choix d'un personnage humanoïde n'était pas anodin. En effet la latéralisation de ce dernier (il a une droite, une gauche, un devant et un derrière) est naturelle et permet un codage relatif de la position des objets par rapport à lui. Ce codage relatif reste utilisable et compréhensible par les opérateurs qu'ils partagent ou non le même point de vue.

\footnotetext{
${ }^{1}$ Un tetramino est une figure géométrique composée de quatre cubes, chacun ayant au moins un côté complètement partagé avec un autre cube.
}

\section{Dispositif Expérimental}

Après la lecture des instructions, chaque participant d'un binôme était installé à la même distance face à un écran LCD 17". Ils tenaient chacun une manette de jeu dans les mains. Les deux participants étaient séparés par un rideau les empêchant de voir l'écran de l'autre mais ils pouvaient communiquer verbalement (Figure 3). Avant de commencer la tâche, les participants avaient quelques essais libres pour se familiariser avec l'interface 3D.

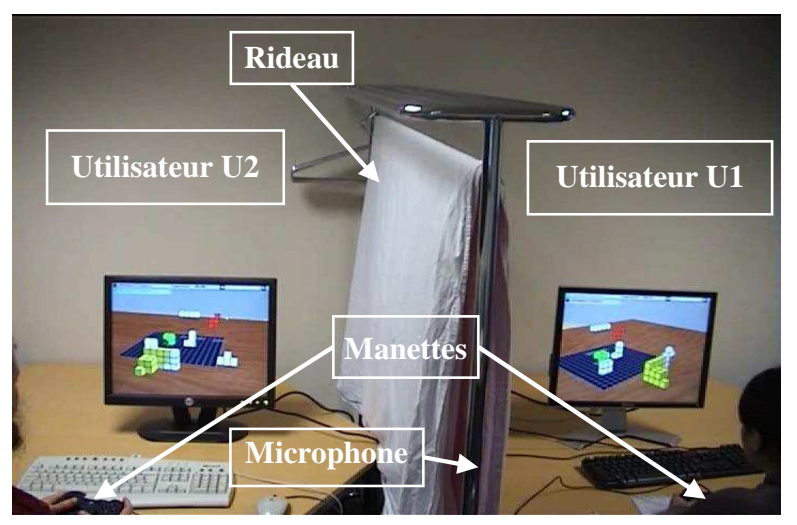

Figure 3 : Dispositif expérimental

\section{Tâche}

Les participants de chaque binôme devaient reconstruire ensemble 5 figures géométriques (modèles, voir figure 4) différentes en utilisant les 6 Tétraminos posés autour. Pour aider les opérateurs à trouver la solution, le premier tétramino était correctement placé au début de la tâche.

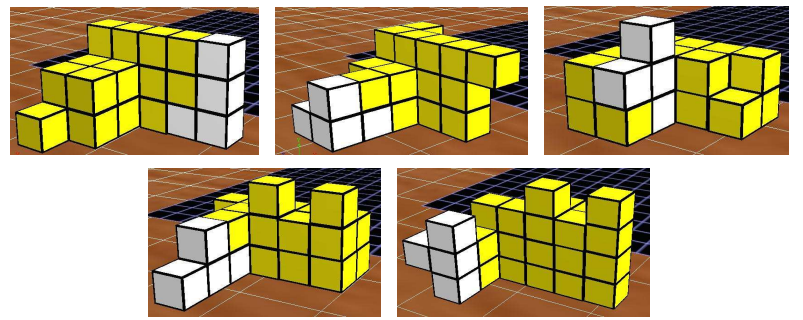

Figure 4 : Les 5 modèles à reproduire

Les actions des participants sur un tétramino étaient matérialisées par un changement de la couleur de ce dernier (rouge ou vert selon l'opérateur). Aucune limitation du temps n'était imposée. Pour chaque modèle, le point de vue de départ des opérateurs sur la scène était différent. Les opérateurs avaient la possibilité de modifier leurs points de vue comme ils le désiraient en cours de tâche.

\section{Variables}

Les facteurs manipulés étaient :

- la présence/absence d'une référence spatiale fixe (PRSF, A-RSF) représentée par le personnage virtuel.

- Le genre des participants (F, G).

\section{Données Recueillies}

Les actions des opérateurs ont été enregistrées avec leurs coordonnées temporelles. Des enregistrements audiovisuels des expérimentations ont aussi été réalisés. Tous 
les dialogues ont été fidèlement retranscrits. Par la suite, les verbalisations ont été filtrées en éliminant les conversations qui ne concernaient pas la résolution de la tâche. Les données analysées sont présentées dans ce qui suit.

\section{Performance}

- Le temps total pour accomplir la tâche

- Nombre d'erreurs : Nombre de fois où une pièce a été utilisée sans être correctement placée.

\section{Verbalisations}

- Utilisation des 3 systèmes de références (égocentré, exocentré et exocentré relatif),

- Utilisation des pronoms : personnels ("je"/"tu") versus impersonnels ("on" et "nous"),

- Nature des verbalisations : les verbalisations ont été classifiées en 16 catégories (cf. table 1).

\section{Traitements Statistiques}

Les données ont étés analysées à l'aide de deux types de tests statistiques :

- Les fréquences d'observation d'un comportement au sein d'un même groupe (choix des référentiels d'action et utilisation des pronoms) ont été comparées à l'aide du test du $\chi^{2}$.

- Etant donné le faible nombre de participants dans chaque groupe, nous n'avons pas pu effectuer d'ANOVA pour comparer les moyennes et seuls des tests $t$ de Student ont été utilisés.

\section{RESULTATS}

\section{Performances}

Temps. Les résultats ont montré que les garçons ont été plus rapides que les filles $(\mathrm{t}(20) \mathrm{p}=0.002)$ pour reconstruire les 5 modèles mais il n'a pas été observé de différences statistiquement significatives entre les groupes P-RSF et A-RSF (t(20) p=0.658).

Erreurs. Les garçons ont fait moins d'erreurs que les filles mais uniquement dans la condition P-RSF ( $\mathrm{t}(9)$ $\mathrm{p}=0.02$ dans la condition P-RSF et $\mathrm{t}(9) \mathrm{p}=0.75$ dans la condition A-RSF).

\section{Verbalisations}

Systèmes de références. Concernant les différences entre les groupes P-RSF et A-RSF : Les filles privilégiaient l'utilisation de la référence égocentrée et cela d'autant plus dans la condition A-RSF $\left(\chi_{\mathrm{k}-1}^{2}=0.1747\right.$ et $\chi_{\mathrm{k}-1}^{2}=0.7722 ; \mathrm{p}<0.05$, respectivement pour les conditions P-RSF et A-RSF ; Figure 5-a).

Les garçons utilisaient davantage les références exocentrée et exocentrée relative dans la condition P-RSF et la référence égocentrée dans la condition A-RSF $\left(\chi_{\mathrm{k}-1}^{2}=0.03139\right.$ et $\chi_{\mathrm{k}-1}^{2}=0.4898 ; \mathrm{p}<0.05$, respectivement pour les conditions P-RSF et A-RSF ; Figure 5-b).

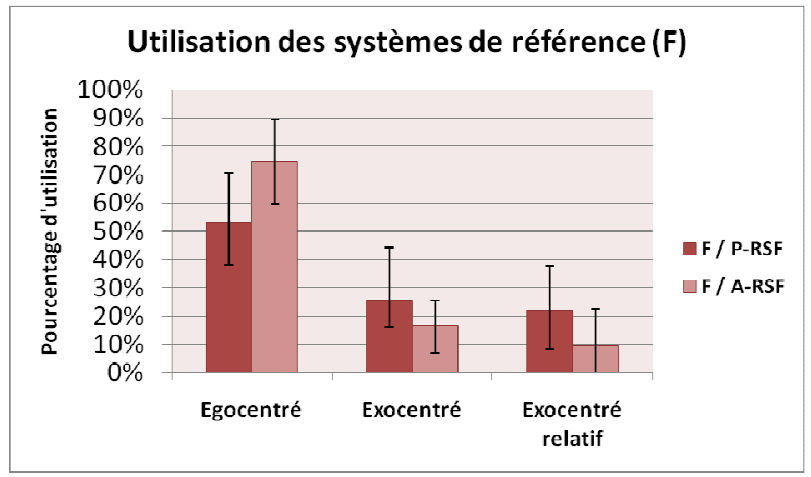

Figure 5-a : Utilisation des sytèmes de référence (Filles)

Concernant les différences entre filles et garçons: Les filles ont utilisé davantage la référence égocentrée que les garçons ( $t(20) \mathrm{p}=0.019$; Figure $5 \mathrm{a}$ et $\mathrm{b})$. Ces derniers ont utilisé plus la référence exocentrée relative que les filles (t(20) p=0.00007; Figure 5 a et b).

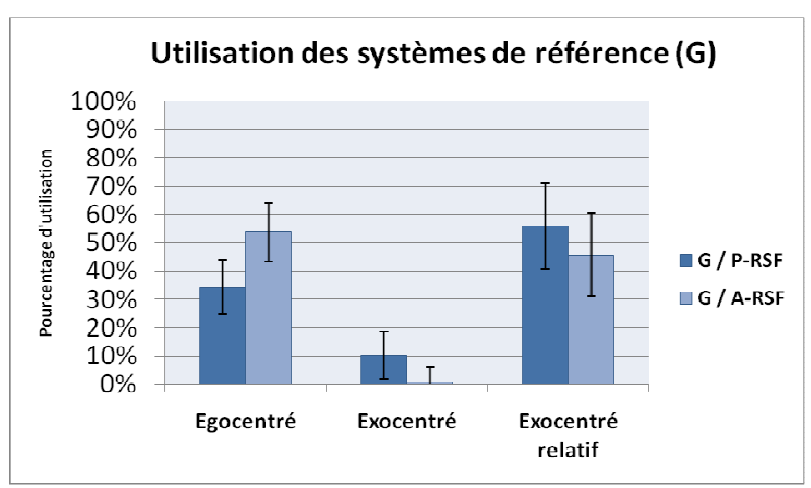

Figure 5-b : Utilisation des systèmes de référence (Garçons)

Utilisation des pronoms. L'analyse a montré que les garçons étaient davantage dans un mode personnel (utilisation des pronoms «je» et «tu ») mais uniquement dans la condition A-RSF $\left(\chi_{\mathrm{k}-1}^{2}=0.0631 ; \mathrm{p}>0.05\right.$ et $\chi_{\mathrm{k}-1}^{2}=0.1094 ; \mathrm{p}<0.05$, respectivement pour les conditions P-RSF et A-RSF). Chez les filles il n'y a pas de différence statistiquement significative entre les pronoms utilisés $\left(\chi_{\mathrm{k}-1}^{2}=0.0403\right.$ et $\chi_{\mathrm{k}-1}^{2}=0.0720 ; \mathrm{p}>0.05$, respectivement pour les conditions P-RSF et A-RSF).

Nature des verbalisations. Concernant les différences entre les filles et les garçons :

Les garçons ont davantage : commenté leur propres actions $(\mathrm{C} 3$, cf. table $1 ; \mathrm{t}(20) \mathrm{p}=0.005)$, fait de commentaires sur la figure en construction (C4; $t(20)$ $\mathrm{p}=0.00001)$, utilisé les déictiques pour montrer leurs actions $(\mathrm{C} 9 ; \mathrm{t}(20) \mathrm{p}=0.015)$ et se sont partagé davantage les tâches (C16 a; t(20) p=0.067 et $\mathrm{C} 16 \mathrm{~b} ; \mathrm{t}(20)$ $\mathrm{p}=0.044$ ) que les filles (Figure $6 \mathrm{a}$ et $\mathrm{b}$ ).

Les filles ont davantage: fait de commentaires sur l'action de leur partenaire $(\mathrm{C} 2 ; \mathrm{t}(20) \mathrm{p}=0.027)$, eu d'incompréhensions explicites $(\mathrm{C} 10 ; \mathrm{t}(20) \mathrm{p}=0.043)$ et fait de réflexions en amont sur la solution (C15a; $\mathrm{t}(20)$ $\mathrm{p}=0.0002$ ) que les garçons (Figure $6 \mathrm{a}$ et $\mathrm{b}$ ). 


\begin{tabular}{|c|c|}
\hline $\mathbf{N}^{\circ}$ & Explication/exemple \\
\hline C1 & $\begin{array}{l}\text { Descriptions des intentions d'actions («je vais } \\
\text { mettre la barre au dessus du carré! ») }\end{array}$ \\
\hline C2 & $\begin{array}{l}\text { Commentaire/question sur une action du partenaire } \\
\text { («A mon avis c'est pas juste ce que tu as fais ») }\end{array}$ \\
\hline $\mathbf{C 3}$ & $\begin{array}{l}\text { Commentaire/question sur sa propre action («je me } \\
\text { suis trompé en faisant ça? ») }\end{array}$ \\
\hline C4 & $\begin{array}{l}\text { Commentaire/question sur la figure («je crois que } \\
\text { les deux premières sont bien placées...») }\end{array}$ \\
\hline C5 & $\begin{array}{l}\text { Approbation de l'action du partenaire («Oui! je } \\
\text { suis d'accord») }\end{array}$ \\
\hline C6 & $\begin{array}{l}\text { Indice de réflexion individuelle (« Attends ! je réflé- } \\
\text { chie...») }\end{array}$ \\
\hline C7 & Difficulté ( «je sais pas ce qu'il faut faire.. ») \\
\hline C8 & $\begin{array}{l}\text { Demande d'approbation sur son action («est ce que } \\
\text { tu es d'accord?») }\end{array}$ \\
\hline C9 & $\begin{array}{l}\text { Déictiques qui servent à monter son action au parte- } \\
\text { naire et qui accompagnent la réalisation concrète de } \\
\text { cette action («mettre ça ici !») }\end{array}$ \\
\hline C10 & $\begin{array}{l}\text { Incompréhensions explicites : aussi bien sur les in- } \\
\text { tentions d'action que sur la disposition des pièces } \\
\text { («je ne comprends pas ce que tu fais! ») }\end{array}$ \\
\hline C11 & $\begin{array}{l}\text { Leadership et verbalisations qui servent à diriger le } \\
\text { partenaire («déplaces ta figure à droite ») }\end{array}$ \\
\hline C12 & $\begin{array}{l}\text { Informations sur les points de vue («moi je suis en } \\
\text { face du bonhomme... ») }\end{array}$ \\
\hline C13 & $\begin{array}{l}\text { Référenciation des pièces («je suis sur la grande } \\
\text { barre ») }\end{array}$ \\
\hline C14 & $\begin{array}{l}\text { Partage des indices de compréhensions («oui je } \\
\text { comprend ce que tu veux dire ») }\end{array}$ \\
\hline C15 & $\begin{array}{l}\text { Stratégies : } \\
\text { a- réflexions en amont sur la solution («on doit pla- } \\
\text { cer cette pièce en premier après ça sera facile »); } \\
\text { b- une stratégie d'essais-erreurs ( «on essaye et on } \\
\text { verra bien si ça marche ») }\end{array}$ \\
\hline C16 & $\begin{array}{l}\text { Répartition des tâches: } \\
\text { a- répartition asymétrique (laisser faire le partenaire) } \\
\text { («je te laisse faire je vois que tu es bien partie »); } \\
\text { b- répartition symétrique (actions simultanés) («toi } \\
\text { tu prends le carré, moi la barre ») }\end{array}$ \\
\hline
\end{tabular}

Table 1 : Les différentes catégories des verbalisations

Concernant les différences entre groupes P-RSF et A-RSF : Dans la condition A-RSF les participants donnent plus d'indices de réflexion individuelle que dans la condition P-RSF $(\mathrm{t}(20) \mathrm{p}=0.048)$. Par ailleurs, la présence de la RSF à une influence variable selon le genre :

Pour les filles, aucune différence statistiquement significative n'a été observée entre les conditions P-RSF et A-RSF (Figure 6-a). A l'inverse, pour les garçons nous observons deux profils distincts :

Les garçons dans la condition P-RSF ont davantage : décrit leurs intentions d'actions $(\mathrm{C} 1 ; \mathrm{t}(10) \mathrm{p}=0.011)$, approuvé les actions de leur partenaire $(\mathrm{C} 5 ; \mathrm{t}(10) \mathrm{p}=0.032)$ et se sont davantage repartis les tâches d'une manière symétrique $(\mathrm{C} 16 \mathrm{a} ; \mathrm{t}(10) \mathrm{p}=0.024)$ que les garçons dans la condition A-RSF (Figure 6-b).

Les garçons dans la condition A-RSF ont davantage: pris le leadership pour diriger leur partenaire (C11; $\mathrm{t}(10) \mathrm{p}=0.028)$ et se sont davantage répartis les tâches d'une façon asymétrique (C16-b ; t (10) p=0.031) que les garçons dans la condition P-RSF (Figure 6-b).

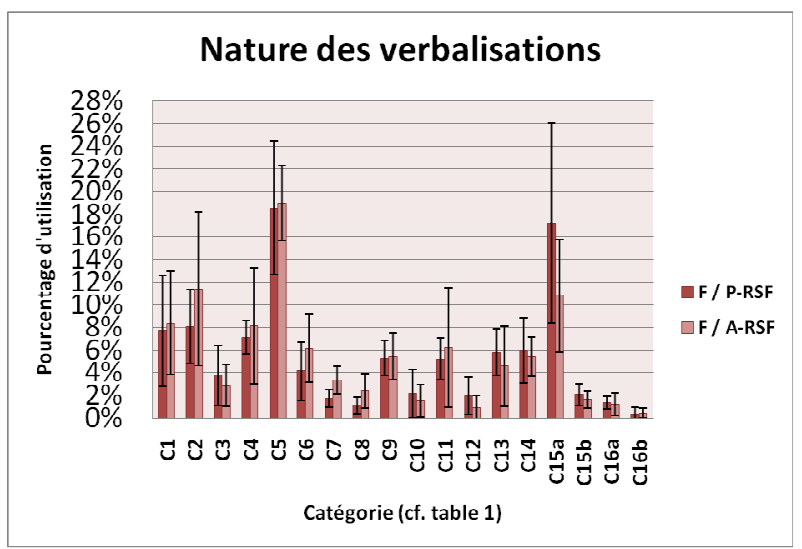

Figure 6-a : Nature des vérbalisations pour lefilles

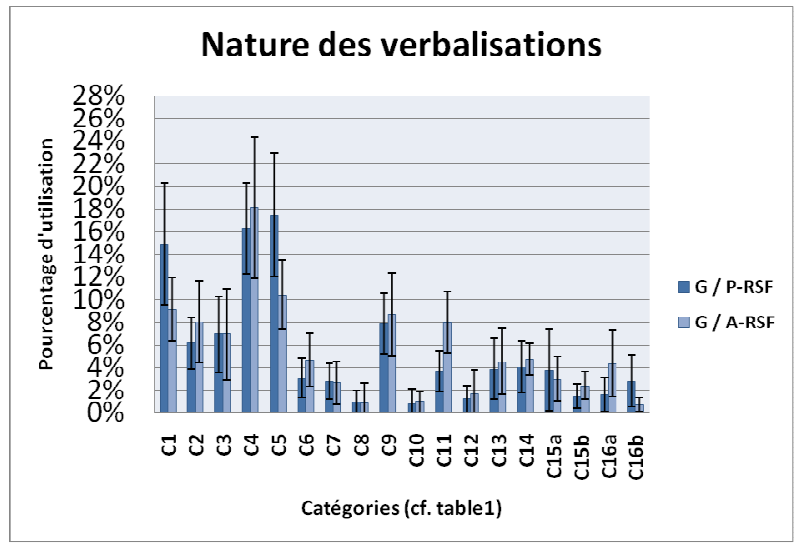

Figure 6-b : Nature des verbalisations pour les garçons

\section{DISCUSSION}

Les résultats montrent que les garçons ont été plus rapides pour la réalisation de la tâche que les filles. L'augmentation du temps pour les filles est accompagnée d'une augmentation du nombre d'erreurs. Ceci montre que les filles ont eu davantage de difficulté à réaliser la tâche. Cette difficulté peut être liée à leur utilisation du système de référence égocentré. Cet argument est cohérent avec les résultats observés chez les garçons. En effet ces derniers ont fait davantage d'erreurs dans la condition A-RSF correspondant à la condition où ils ont davantage utilisé le système de référence égocentré.

\section{Influence de la RSF}

Les résultats montrent que les garçons ont été influencés par la présence de la RSF. En effet ils sont passés de l'utilisation majoritaire d'un système égocentré en l'absence de la RSF à l'utilisation majoritaire des systèmes exocentré et exocentré relatif en présence de la RSF. Cependant les filles semblent moins sensibles à la présence de la RSF. En effet, elles ont gardé leur préférence d'utilisation majoritaire du système égocentré, même si elles ont augmenté leur utilisation des systèmes exocentré et exocentré relatif en présence du personnage. L'hypothèse $\mathrm{H} 1$ est donc vérifiée uniquement pour les 
binômes masculins. Par ailleurs, ceci confirme l'hypothèse $\mathrm{H} 2$.

D'un autre coté, certains utilisateurs ont tenté d'utiliser d'autres objets de l'EV comme référence spatiale. Cependant, ceci a induit certains problèmes de compréhension car ces objets n'étaient pas latéralisés. Voici un extrait d'une conversation qui illustre ces problèmes :

Op1 : «Mets-toi face au modèle jaune... »;

Op2 : "Euh, oui mais je ne sais pas...on est tout le temps face au modèle!»

\section{Construction du RC}

Concernant la construction d'un RC (hypothèse $\mathrm{H} 2$ ), on s'est intéressé aux conversations sur les actions. Ainsi deux cas peuvent être discutés. Les garçons, en présence de la RSF, ont décrit plus leurs intentions d'actions, ont eu plus de retours positifs de leur partenaire sur ces actions (approbations) et ils ont agit plus souvent en parallèle. Les garçons en l'absence de cette RSF ont eu davantage de retours négatifs (commentaires et interrogations) et ont tenté le plus souvent d'utiliser leur partenaire pour faire les actions. Ces résultats suggèrent un RC plus efficient pour les garçons en présence de la RSF. En effet, une spécification d'actions plus facile et des retours positifs peuvent être interprétés comme étant des signes d'entente entre les partenaires. Ainsi ils pouvaient agir en parallèle puisque ils étaient d'accord à l'avance sur les actions à faire (une solution commune). D'un autre coté, les commentaires et interrogations sur les actions du partenaire peuvent être considérés comme des signes de mésentente entre les partenaires. C'est pourquoi certains participants prenaient l'initiative en dirigeant leur partenaire selon leurs propres solutions (instauration d'un leadership). Ceci explique aussi la répartition asymétrique des tâches.

Pour les filles, il est difficile de dire si elles avaient un RC plus efficient dans une condition plus que dans une autre car on observe peu de différences entre les binômes. Ceci implique la validation de l'hypothèse $\mathrm{H} 3$ pour les binômes masculins et la nécessité de faire davantage d'investigations pour les binômes féminins. Par ailleurs, On a observé que tous les participants ont essayé de contourner le problème de description des actions en profitant des indices visuels caractérisant l'interface. En effet, l'utilisation des déictiques montre qu'ils se sont servis des indices sur les activités (changement des couleurs des tétraminos) pour montrer leurs actions et attirer l'attention de leur partenaire. Ainsi les garçons (dans les conditions P-RSF et A-RSF) en utilisant plus de déictiques pour se montrer leurs actions ont eu moins d'incompréhensions explicites que les filles.

\section{Profils de Collaboration}

Pour les garçons, on peut extraire deux profils de collaboration dépendants de l'utilisation de la RSF :
Profil négociateur. En présence d'une RSF, les garçons ont un profil de groupe avec davantage de : discussions sur leurs intentions d'actions, d'approbation des actions du partenaire que de commentaires et interrogations sur celles-ci, d'actions parallèles et moins de tentatives pour diriger leur partenaire. Cela est associé à une utilisation majoritaire des références exocentrée et exocentrée relative et des pronoms impersonnels.

Profil individualiste. En l'absence de la RSF, ils sont dans un mode plus «personnel» avec moins de spécifications d'actions, davantage d'indices de réflexion individuelle, une répartition asymétrique des tâches, autant de commentaires et d'interrogations sur les actions du partenaire que d'approbations de celles-ci et plus de tentatives de diriger le partenaire (instauration d'un leadership). Ceci est associé à une plus grande utilisation du système de référence égocentré et des pronoms

Profil féminin. Les filles quant à elles sont moins sensibles à la présence de la RSF. Néanmoins le fait qu'elles aient moins partagé leurs réflexions en amont dans la condition A-RSF alors qu'elles ont davantage commenté les actions de leur partenaire et ont donné plus d'indices de réflexion individuelle laisse pensé que l'absence de la RSF diminue les efforts de réflexion commune sur la figure à construire. Cependant, la grande variabilité des résultats empêche l'extraction d'un profil féminin précis.

\section{Bilan}

En résumé, ces résultats ont deux implications : d'abord, la différence observée entre les filles et les garçons quant aux référentiels d'action privilégiés (égocentré pour les filles/exocentré et relatif pour les garçons) et leur sensibilité à la présence de la RSF. La deuxième implication concerne l'influence des référentiels d'action sur la nature de la collaboration qui s'instaure entre les participants masculins (formulé dans l'hypothèse H4). Ainsi lorsque la référence égocentrée est utilisée, les participants adoptent davantage un style de collaboration personnel caractérisé par une utilisation accrue de l'autre (leadership). Lorsque les références exocentrée et relative sont privilégiées les participants tendent vers un profil de groupe avec davantage de spécifications des actions et de réflexion commune en amont ce qui confirme l'hypothèse $\mathrm{H} 4$ pour les binômes masculins. Néanmoins, les profils extraits n'ont pas influencé le succès de la tâche. Cela est dû notamment à l'utilisation de stratégies de compensation visuelle (montrer les actions) et verbales (diriger le partenaire) pour palier aux problèmes de compréhension.

\section{CONCLUSION}

Cette étude s'inscrit dans un travail visant à fournir des recommandations pour la conception d'EV supportant les activités collaboratives. Les résultats ont montré une différence entre filles et garçons dans leur façon de collaborer pour une tâche de reconstruction de figures 3D. 
Les résultats ont également montré que la présence d'indices contextuels (la RSF) dans l'EV a influencé leur manière de collaborer. Ainsi l'ajout du personnage virtuel comme indice contextuel dans l'EV a un effet positif sur la collaboration dans le cas d'utilisateurs masculins. En effet il améliore la construction du RC entre les utilisateurs et en particulier la coordination des actions.

Pour être efficace dans la construction d'un RC dans une scène virtuelle, l'indice contextuel doit selon nous respecter certaines caractéristiques :

- Il doit être latéralisé de façon à permettre un codage exocentré de la position des objets par rapport à lui ;

- Il doit être immobile afin d'éviter un changement dans le codage exocentré en cours de tâche ;

- Il doit être facile à nommer et facilement identifiable par rapport aux autres objets virtuels afin d'éviter les ambigüités ;

- Il doit être visible par tous les utilisateurs qui collaborent à un instant donné pour que la référence exocentrée soit accessible à tous simultanément.

Enfin, l'utilisation d'un personnage anthropomorphique comme référence spatiale peut avoir des effets sur la présence des utilisateurs dans l'EV. En effet, en l'absence d'une représentation des utilisateurs dans l'EV, nous avons observé que certains d'entre eux se projetaient spontanément dans ce personnage. Ainsi ils l'utilisaient comme leur représentation commune. Cette représentation leur permettait de spécifier leurs actions dans un système de référence «égocentré projeté sur l'avatar» unique et partagé.

Dans nos prochaines études, nous introduirons :

(a) Des tâches où le succès est directement lié à la collaboration (une tâche de comanipulation par exemple) pour pouvoir évaluer l'influence des profils de collaboration sur les performances.

(b) La dimension haptique comme moyen supplémentaire de communication dans L'EV. L'enjeu sera de savoir s'il est possible d'améliorer la compréhension entre les opérateurs en leur donnant plus de moyens pour renseigner leur interlocuteur sur leurs activités.

Les résultats de ces études pourront servir à concevoir des EVC facilitant la collaboration.

\section{REMERCIMENTS}

Cette recherche a été en partie financée par le projet Interacteurs (CRE $\left.\mathrm{N}^{\circ} 43230501\right)$ avec Orange Labs. Nous tenons également à remercier les étudiants de Nantes ayant accepté de participer à l'expérience.

\section{BIBLIOGRAPHIE}

1. Axelsson, AS. Abelin, Å. \& Schroeder, R. Communication in Virtual Environments: Establishing Common Ground for a Collaborative Spatial Task.
The 6th International Workshop on Presence, Aalborg, Denmark, October 6-8. 2003.

2. Blouin, J., Bard, C., Teasdale, N., Paillard, J., Fleury, M., Forget, R., \& Lamarre, Y. (1993). Reference systems for coding spatial information in normal subjects and a deafferented patient. Experimental Brain Research, 93, pp. 324-331.

3. Bridgeman, B., (1999). Separate representations of visual space for perception and visually guided behavior. In G. Aschersleben, T. Bachmann \& J. Müsseler (Eds). Cognitive Contribution to the Perception of Spatial and Temporal Events (pp. 3-18).

4. Churchil, E. F. Snowdon, D. In Collaboratif Virtual Environnement 1998. SIGGROUP Bulletin 1998 vol $19 \mathrm{~N}^{\circ} 2$.

5. Clark, H.C. \& Brennan, S. (1991) Grounding in communication. In L. B. Resnick, J. Levine \& S. D. Teasley (Eds.), Perspectives on socially shared cognition. Washington: APA Books.

6. Dillenbourg, P. (1999). What do you mean by collaborative learning? In Dillenbourg (Ed.), Collaborative Learning: Cognitive and Computational Approaches: Elsevier Science/Pergamon.

7. Harrison, S. \& Dourish, P. (1996). Re-place-ing space: the roles of place and space in collaborative systems. In proceedings of CSCW' 96.

8. Hindmarsh, J., Fraser, M., Heath, C., Benford, S. \& Greenhalg, C. (1998). Fragmented Interaction: Establishing mutual orientation in virtual environments. In CSCW'98, 217-226.

9. Kimura, D. (2001). Cerveau d'homme et cerveau de femme? Les aptitudes spatiales (pp. 78-79). Paris: Odile Jacob.

10. Kraut, R. E., Fussell, S. R. \& Siegel, J. (2003). Visual information as a conversational resource in collaborative physical tasks. HCI, 18, 13-49. 2003.

11. Loiselet, A. \& Hoc, J.M. La gestion des interférences et du référentiel commun dans la coopération: implications pour la conception. Psychologie Française 2001; 46, pp. 167-179.

12. Roberts R. J. Jr. \& Aman, C. (1993). Developmental Differences in Giving Directions: Spatial Frames of Reference and Mental Rotation. Child Development 64 (4): 1258-70.

13. Roschelle, J. \& Teasley, S.D. (1995). Construction of shared knowledge in collaborative problem solving. In C. O'Malley (Ed.), CSCL. (69-97).

14.Spante, M., Schroeder, R. \& Axelsson, AS. (2004). How Putting Yourself into the Other Person's Virtual Shoes Enhances Collaboration. Proceeding of the 7th International Workshop on Presence, Valencia, Spain-, pp. 190-196. 